\title{
Assessing Cardiovascular Comorbidities in Sleep Apnea Patients Using $\mathrm{SpO}_{2}$
}

\author{
Margot Deviaene ${ }^{1,2}$, Carolina Varon ${ }^{1,2}$, Dries Testelmans ${ }^{3}$, Bertien Buyse ${ }^{3}$, Sabine Van Huffel ${ }^{1,2}$ \\ ${ }^{1}$ KU Leuven, Department of Electrical Engineering (ESAT), STADIUS Center for Dynamical \\ Systems, Signal Processing and Data Analytics, Leuven, Belgium \\ ${ }^{2}$ Imec, Leuven, Belgium \\ ${ }^{3}$ UZ Leuven, Department of Pneumology, Leuven, Belgium
}

\begin{abstract}
Several studies have demonstrated the relationship between Obstructive Sleep Apnea Syndrome (OSAS) and cardiovascular comorbidities. It is even suggested that timely OSAS treatment can prevent the development of such comorbidities. Hence, it is important to identify the patients with a high risk for cardiovascular comorbidities and prioritize their treatment. This study investigates if the blood oxygen saturation $\left(\mathrm{SpO}_{2}\right)$ signal could be used to assess the cardiovascular status of the patient. This on its turn can improve the phenotyping of OSAS patients.

$\mathrm{SpO}_{2}$ signals from 100 OSAS patients, of which half have a known cardiovascular comorbidity, are investigated. The individual oxygen desaturations are extracted and these desaturations are classified as caused by a respiratory event or not. This classification is then used to compute patient averaged features of apneic and non-apneic desaturations. The most discriminative features to differentiate between patients with and without cardiac comorbidity are selected. Using these, a Leastsquares Support Vector Machine (LS-SVM) classifier reached an accuracy of $76.7 \%$ on separating test set patients according to their cardiac comorbidity status.

These results suggest that the analysis of the $\mathrm{SpO}_{2}$ signal has an added value in the assessment of the cardiovascular risk of OSAS patients.
\end{abstract}

\section{Introduction}

Obstructive Sleep Apnea Syndrome (OSAS) is the most common sleep related breathing disorder. It affects around $13 \%$ of men and $6 \%$ of women, among adults between 30 and 70 years old [1]. The disease presents itself as complete or partial cessations of breathing during the night due to blockage of the upper airways or loss of respiratory drive. These events usually result in hypoxia and/or arousal, which trigger the restoration of normal breathing.

The hypoxia, arousals, and negative intrathoracic pressure swings due to respiratory events deteriorate the cardiovascular system via different pathways. Changes in both parasympathetic and sympathetic activity have been recorded, as well as a decreased cardiac output, inflammation and endothelial dysfunction [2].

These adverse effects cause patients with OSAS to have an increased risk of developing hypertension, diabetes and cardiovascular events such as myocardial infarction and stroke, even when adjusted for confounding factors such as obesity [2]. An observational study by Marin et al., showed that patients with untreated severe OSAS have an adjusted odds ratio of 3.17 for developing non-fatal cardiovascular events with respect to healthy subjects [3]. The patient group treated with continuous positive airway pressure (CPAP), however, had no significant increase in risk. These results suggest that treatment with CPAP can decrease the risk of developing cardiovascular events.

Therefore, it is important to identify high risk patients in order to avert severe cardiovascular comorbidities by timely OSAS treatment. Nowadays OSAS severity is defined by the apnea hypopnea index (AHI), which corresponds to the amount of respiratory events per hour of sleep. It has been shown, however, that the AHI is not a very good predictor for cardiovascular events, when adjusted for confounding factors [4]. In contrast, the time spent with oxygen saturation below $90 \%$, proved to be a significant predictor. This indicates that the $\mathrm{SpO}_{2}$ signal contains useful information with respect to the cardiovascular comorbidity of the patient.

In this study, parameters of individual oxygen desaturations due to respiratory events will be compared for OSAS patients with and without cardiovascular comorbidity. Using the most discriminative features, an indication of the presence of cardiovascular comorbidities is automatically computed.

\section{Methodology}

An overview of the methodology used in this study can be found in Figure 1. The different steps will be explained in the following paragraphs, starting with a description of the used dataset. 


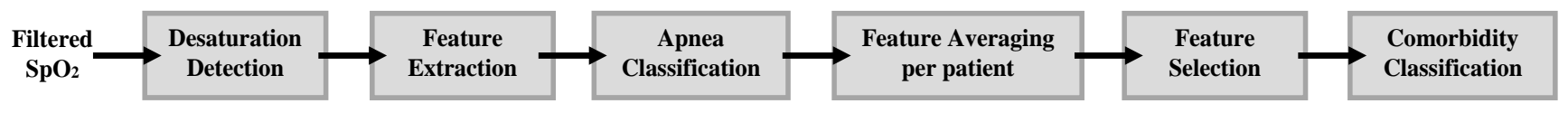

Figure 1. Overview of the proposed algorithm to create a cardiovascular comorbidity detector.

\subsection{Data}

In this study, $\mathrm{SpO}_{2}$ signals extracted from polysomnography (PSG) recordings of patients referred to the University Hospitals Leuven are analysed. The $\mathrm{SpO}_{2}$ signals were recorded using a Nonin oximeter and sampled at $500 \mathrm{~Hz}$. The PSG data was annotated by sleep specialists according to the AASM 2012 scoring rules [5]. Additionally, an assessment of cardiovascular comorbidities was made for each patient. The presence of hypertension, hyperlipidaemia and diabetes were listed as well as previous occurrences of myocardial infarction or stroke. Patients with an AHI larger than 15 were selected from the hospital's database. Subjects with at least one of the above cardiovascular comorbidities were matched for age, gender, body mass index (BMI) and smoking status with a patient without any comorbidity.

A dataset of 100 patients was collected, including 78 men and 22 women with an average age of $48 \pm 10.8$ years and a BMI of $30 \pm 4.5 \mathrm{~kg} / \mathrm{m}^{2} .24 \%$ of the patients smoked at the time of the PSG and the average AHI is $41.3 \pm 22.0$. Hyperlipidaemia and hypertension were the most common comorbidities, present in, respectively, 46 and 40 subjects, 5 patients suffered from diabetes, 4 patients had a myocardial infarction and 2 a stroke.

This dataset is split into a training set of 70 patients and a test set of 30 patients, using the patient characteristics and making sure half of the patients in each set have a comorbidity.

\section{2. $\quad \mathrm{SpO}_{2}$ preprocessing}

The aim of the study is to detect changes in the $\mathrm{SpO}_{2}$ desaturations due to cardiovascular comorbidities. Therefore, the individual desaturations need to be extracted from the $\mathrm{SpO}_{2}$ signal. Before doing so, zero level artefacts caused by sensor disconnections are detected when the signal drops below $50 \%$. An interval around the drop is replaced by linear interpolation. Sharp changes and ripples due to oversampling are corrected by means of a moving average filter of 3 seconds. Afterwards the signal is down sampled to $1 \mathrm{~Hz}$.

The desaturation events are detected as peaks in the derivative of this filtered signal. Each event consists of a desaturation part where the $\mathrm{SpO}_{2}$ drops, possibly followed by a stable $\mathrm{SpO}_{2}$ period, and ending with the resaturation where the $\mathrm{SpO}_{2}$ tends to go back to baseline level. The start and end points of the desaturation and the following resaturation are used to segment the signals. Events with a drop of more than $1 \%$ in $\mathrm{SpO}_{2}$ and a total duration of less than $120 \mathrm{~s}$ are taken into account.

In addition, the $\mathrm{SpO}_{2}$ baseline is defined as the $95^{\text {th }}$ percentile from the last minute of artefact and desaturation free signal.

\subsection{Feature extraction}

A total of 143 features and their logarithmic transformation were extracted from all detected events. Simple time-domain features are extracted such as the amplitudes, lengths and slopes of the desaturation and resaturation. Furthermore, the obstruction severity, defined as the length of the event multiplied by the area under baseline is extracted [6]. Additionally, the time and area that the $\mathrm{SpO}_{2}$ spends under baseline or 2,3 or $4 \%$ below baseline during the event is computed $[7,8]$.

In addition, the minimum, maximum, mean, median and variance are extracted from the $\mathrm{SpO}_{2}$ signal and its first, second and third order derivative. These features are extracted 3 times, for the complete event, the desaturation and the resaturation part. Moreover, deviations of the minima and maxima from the above mentioned medians and means are computed, as Koley et al. did for the detection of respiratory events in the $\mathrm{SpO}_{2}$ signal [8].

The last group of features takes into account the fact that patients often have episodes where a sequence of respiratory events occur in close succession. To assess these quasi-periodicities, a window of five minutes around the detected desaturation is analyzed. The autocorrelation (AC) function of this segment is computed and the relative amplitude and position of the first peak, representing the delay between respiratory events, are extracted. Another way to analyze these segments is by using Phase Rectified Signal Averaging (PRSA), which detects quasiperiodicities in non-stationary data [9]. The PRSA curve is computed as the average of all 10 second downward or upward fragments in the five minute segment, representing respectively the averaged desaturation and resaturation. From this curve, the maximum amplitude difference and slope over the entire curve are extracted.

\subsection{Respiratory event classification}

Using these features, a classifier is built to separate the detected desaturations caused by an annotated respiratory event and desaturations not related to respiratory events. Using feature selection techniques, the six most discriminative features are selected. These contain the 
duration and area under the baseline of the desaturation part, the time spent under $2 \%$ below the baseline, the PRSA amplitude for desaturations and resaturations and the relative amplitude of the first AC peak.

With these six features, a Least Squares Support Vector Machine (LS-SVM) classifier [10], using an RBF kernel is built based on data of the 70 training patients.

\subsection{Cardiac comorbidity classification}

The estimated class labels obtained by the respiratory event classification are used to separate the desaturations in two groups, one containing the desaturations caused by respiratory events (apneic), the second one containing all other desaturations (non-apneic). Patient averaged features are computed for each group, once considering all desaturations, and a second time only considering desaturations with a drop from baseline larger than $3 \%$.

These patient averaged features are then used to classify the patients as having a cardiovascular comorbidity or not. The most discriminative features need to be selected, based on the data of the 70 training patients, before a classifier can be trained. A first selection is made based on statistical significance, features with a p-value above 0.05 , computed using the Wilcoxon rank sum test, are discarded. Next highly correlated features are removed, feature couples with a correlation larger than 0.5 are inspected, starting with the highest correlation. For each couple only the feature with the highest F-test score, representing the between group variability on the within group variability, is kept. The last step is to perform a backwards wrapper on the remaining features, using an RBF LS-SVM classifier and based on the Leave One Out Cross Validation (LOO$\mathrm{CV})$ cost.

Using the selected features, an RBF LS-SVM classifier is trained using LOO-CV, to separate OSAS patients with a cardiovascular comorbidity from OSAS patients without comorbidity.

\section{Results and discussion}

The respiratory event classifier reached an accuracy of $80.0 \%$ on the independent test set of 30 patients with a sensitivity of $86.0 \%$ and a specificity of $72.7 \%$. This is a promising result, taking into account the facts that no sleep segmentation was performed and that the classification is only based on the $\mathrm{SpO}_{2}$ signal. This signal is easy to acquire in a home environment and is unobtrusive for the patient, in contrast to the full PSG.

The cardiac comorbidity feature selection resulted in the selection of seven features, which are plotted in Figure 2 for patients without and with cardiovascular comorbidities. The feature set contains the logarithm of four features extracted from respiratory events with an $\mathrm{SpO}_{2}$ drop of more than $3 \%$ from the baseline: the mean and median of the derivative, the median of the second order derivative of the desaturation part and the deviation of the median value from the maximal value. The logarithms of three features were extracted from desaturations not associated with respiratory events: the area under baseline and the PRSA downwards slope, averaged over all desaturations, the deviation between the mean and maximum was taken over drops larger than $3 \%$.

The performance of the resulting cardiovascular comorbidity classifier on the training set and the independent test set can be seen in Table 1. An accuracy of $76.7 \%$ is achieved on the independent test set, with a sensitivity of $80 \%$ and a specificity of $73.3 \%$.

Table 1. Performance of the cardiovascular comorbidity classifier on the training and independent test set.

\begin{tabular}{l|cccccc}
\hline Data & Acc. & Se. & Sp. & PPV & AUC & $\begin{array}{c}\text { Cohen } \\
\kappa\end{array}$ \\
\hline Train & 97.1 & 97.1 & 97.1 & 97.1 & 0.99 & 94.3 \\
Test & 76.7 & 80.0 & 73.3 & 75.0 & 0.76 & 53.3 \\
\hline
\end{tabular}

When analysing the features in Figure 2, one can see that the two classes are statistically different for all features on the complete dataset. If only the test set is considered, however, only features 3 and 5 have a significant difference. These features imply that OSAS patients with a cardiovascular comorbidity have a lower $2^{\text {nd }}$ order derivative of the $\mathrm{SpO}_{2}$ signal during desaturation and larger areas under the $\mathrm{SpO}_{2}$ baseline during non-apneic desaturations.

The lower second order derivative values imply a slower change in slopes of the $\mathrm{SpO}_{2}$ signal after a loss of oxygen supply due to a respiratory event. This effect could be due to a deterioration of the cardiovascular system, which results in a delayed oxygen desaturation in the blood.

The fact that patients with cardiovascular comorbidities have larger areas under the $\mathrm{SpO}_{2}$ baseline is in agreement with theories that more severe hypoxias have a higher probability to cause damage to the cardiovascular system [6,7]. Features 4 and 7 can also be seen as a hypoxia severity parameter, they represent the amplitude difference between the median, respectively the mean, and the maximum $\mathrm{SpO}_{2}$. These values increase if a patient has a cardiovascular comorbidity.

The subjects with a cardiovascular comorbidity that were misclassified as having no comorbidity all had hypertension and/or hyperlipidaemia. All patients with more severe cardiovascular comorbidities were correctly classified. It should be noted that for this study no information was available on the duration and severity of the particular cardiovascular disease of the patient. This information might have been interesting in order to see if the obtained features would differ between progressing disease stages. Moreover, patients without cardiac 


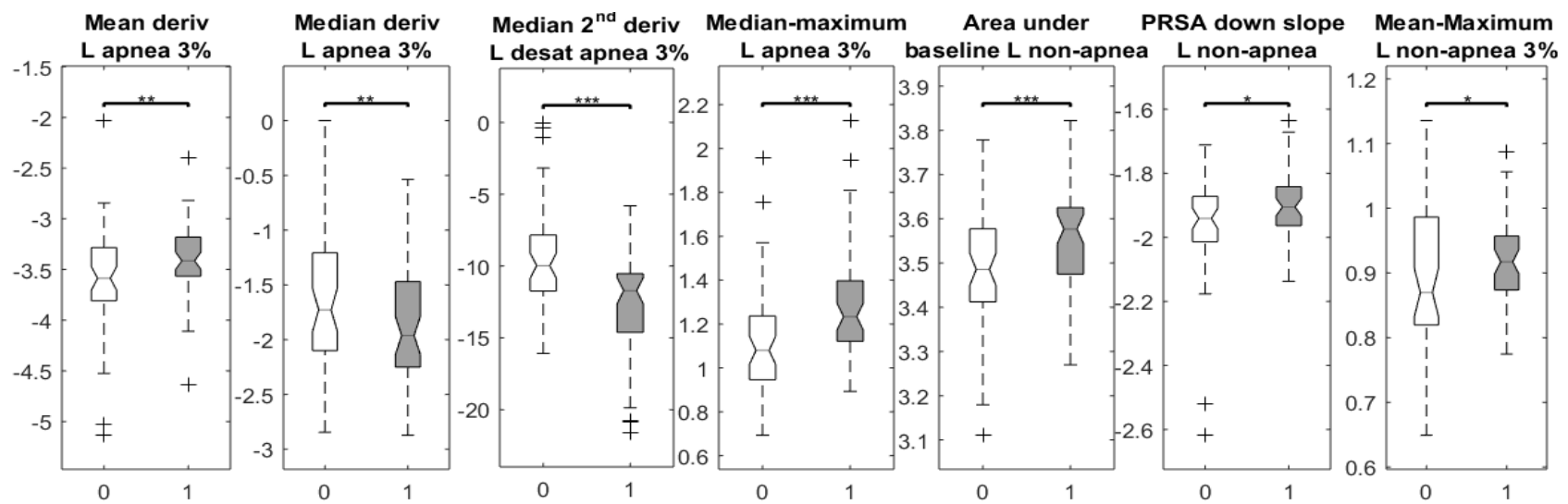

Figure 2. Features of comorbidity classification for patients without (0) and with cardiovascular comorbidity (1). Statistical significance between groups, Wilcoxon rank sum test: * $\mathrm{p} \leq 0.1 * * \mathrm{p} \leq 0.05 * * * \mathrm{p} \leq 0.01$.

comorbidity could already be in a late stage of development of cardiovascular disease, but without any observable symptoms yet.

The current study is performed on a limited dataset with only 30 patients used for testing. Therefore, to generalize these findings, a larger dataset should be considered. In order to move from assessing cardiovascular status to predicting the cardiovascular risk of the patient, follow-up studies should be considered to see which OSAS patients develop these comorbidities over time.

In this study only patients with an AHI larger than 15 were considered, in the future, it should be studied if the same effects in the $\mathrm{SpO}_{2}$ signal can be seen in patients with a lower AHI. These patients are nowadays not treated with CPAP, but maybe for some of these patients CPAP treatment would decrease their risk of developing cardiovascular comorbidities.

\section{Conclusion}

The findings in this study suggest that cardiovascular comorbidities in OSAS patients can be detected based on changes in the $\mathrm{SpO}_{2}$ signal. This could be interesting for the phenotyping of OSAS patients and their treatment decisions. Suggestions were made for further studies in order to be able to generalize these results.

\section{Acknowledgements}

This work is supported by: Agentschap voor Innovatie door Wetenschap en Technologie (IWT) Project \#:

SWT 150466 - OSA+. imec funds 2017. imec ICON projects: ICON HBC.2016.0167. European Research Council: The research leading to these results has received funding from the European Research Council under the European Union's Seventh Framework Programme (FP7/2007-2013) / ERC Advanced Grant: BIOTENSORS $\left(n^{\circ} 339804\right)$. This paper reflects only the authors' views and the Union is not liable for any use that may be made of the contained information.

\section{References}

[1] Peppard P et al. Increased prevalence of sleep-disordered breathing in adults. Am J Epidemiology 2013;177.9;100614.

[2] Bradley TD, Floras JS. Obstructive sleep apnoea and its cardiovascular consequences. The Lancet 2009;373;82-93.

[3] Marin JM et al. Long-term cardiovascular outcomes in men with obstructive sleep apnoea-hypopnoea with or without treatment with continuous positive airway pressure: an observational study. The Lancet 2005;365; 1046-53.

[4] Kendzerska T et al. Obstructive sleep apnea and risk of cardiovascular events and all-cause mortality: a decade-long historical cohort study.PLoS medicine 2014;11.2; e1001599.

[5] Berry RB et al. Rules for scoring respiratory events in sleep: update of the 2007 AASM manual for the scoring of sleep and associated events. J Clin Sleep Med 2012;8.5: 597-619.

[6] Kulkas A et al. Novel parameters indicate significant differences in severity of obstructive sleep apnea with patients having similar apnea-hypopnea index. Med Biol Eng Comput 2013;51.6;697-708.

[7] Watanabe E et al. Prognostic importance of novel oxygen desaturation metrics in patients with heart failure and central sleep apnea. Journal of cardiac failure 2017;23.2;131-137.

[8] Koley B et al. On-Line Detection of Apnea/Hypopnea Events Using $\mathrm{SpO}_{2}$ Signal: A Rule-Based Approach Employing Binary Classifier Models. IEEE JBHI 2014;18.1;231-239.

[9] Bauer A. et al. Phase-rectified signal averaging detects quasi-periodicities in non-stationary data. Physica A: Statistical Mechanics and its Applications 2006;364;423434.

[10] Suykens J et al. Least squares support vector machines. World Scientific, 2002.

Address for correspondence:

Margot Deviaene

ESAT/STADIUS/KU Leuven

Kasteelpark Arenberg 10, bus 2446, 3001 Leuven, Belgium.

margot.deviaene@esat.kuleuven.be 